\title{
Barriers and Facilitators for Physical Activity in Already Active Pregnant and Postpartum Women? Findings from a Qualitative Study to Inform the Design of an Intervention for Active Women.
}

Murali Krishnan Perumbakkam Subramanian ( $\square$ m.perumbakkamsubramanian@tees.ac.uk) Teesside University School of Health and Social Care https://orcid.org/0000-0001-8592-6985

Peter Van der Graaf

Teesside University School of Health and Social Care

Rosemary Dawson

Teesside University School of Health and Social Care

Louise Hayes

Newcastle University Institute for Health and Society

Louisa J Ells

Leeds Beckett University

Kunj Sachdeva

All India Institute of Medical Sciences

Liane Azevedo

University of Huddersfield School of Human and Health Sciences

Research article

Keywords: Physical Activity, Already Active, Interventions, Barriers and Facilitators

Posted Date: June 9th, 2020

DOI: https://doi.org/10.21203/rs.3.rs-33568/v1

License: (c) (1) This work is licensed under a Creative Commons Attribution 4.0 International License. Read Full License 


\section{Abstract \\ Background -}

Activity levels decline substantially in both inactive and active women during pregnancy and postpartum. There is limited information on the barriers and facilitators for physical activity participation in active women during this period. The primary aim of this study is to identify the barriers and facilitators for physical activity in already active women during pregnancy and postpartum. We also explore their views on, and requirements for, the development of an intervention to support the maintenance of or increase in physical activity.

\section{Methods}

- Five focus groups, with a total of 19 participants, were conducted. Transcripts were analysed using a thematic analysis approach.

\section{Results}

- Out of the 19 participants, four were postpartum, and 15 were pregnant. Analysing the focus group transcripts, 22 codes were generated and grouped into eight themes: 1-cognizant of physical activity's benefits, 2-sources of advice, 3-reasons to be active during pregnancy, 4-reasons for reducing physical activity levels during pregnancy, 5-barriers to physical activity during and after pregnancy, 6-facilitators of physical activity during and after pregnancy, 7-ideal physical activity intervention, and 8-evaluation support.

\section{Conclusions}

- Among already active pregnant and postpartum women, factors such as pregnancy-related body changes, childcare and lack of targeted activities deter participation in physical activity. A new programme with social and group elements, including both familiar and new activities, and providing 'satisfaction and fun' should be developed to help increase or maintain their activity levels.

\section{Background}

There is good evidence demonstrating the health benefits of being active $(1,2)$. During pregnancy and postpartum physical activity has been shown to prevent several maternal and fetal conditions $(3,4)$. The benefits of physical activity during pregnancy include improving maternal glucose levels (5), reducing the risk of gestational diabetes and impaired glucose tolerance (6) and supporting healthy neonatal birth weight (7). National statistics demonstrate that only around half of all women meet current UK physical activity guidelines (8), with activity levels in pregnant women being even lower $(9,10)$. Studies report that 
pregnant women spend more than half of their time sedentary and do not meet physical activity recommendations $(9,10)$. Even among active women, there is a tendency to reduce physical activity levels during pregnancy, and this pattern of inactivity can persist into the postpartum period and beyond (11-16).

It has been proposed that the barriers and facilitators to being active during and after pregnancy may be different for those women who were active, as opposed to inactive prior to pregnancy (13). However, the evidence exploring the barriers and facilitators to physical activity during pregnancy and postpartum, specifically within previously active women is limited (17). A qualitative study conducted a few years post-pregnancy among already active women to identify their perceptions of physical activity during pregnancy has highlighted that there are barriers they needed to overcome to remain active during pregnancy (17). Despite accomplishing activity, they noticed a decrease in activity levels and a skewing towards alternate activities that provide more enjoyment. In contrast, the present study, engaged with active women who are currently pregnant or in the postpartum period, living in deprived areas of the North East of England. This study is part of a larger project, the Sport England - Helping the active to Stay Active, which has the overarching aim of informing the design of a physical activity intervention for currently active pregnant and postpartum women.

Therefore, the aims of this qualitative study are to 1) investigate the barriers and facilitators to physical activity in women who were active prior to pregnancy and the postpartum period, and 2) explore views on, and requirements for, the development of an intervention to support maintain or increase physical activity levels, with the aim of preventing the decrease of activity currently observed in this population.

\section{Methods}

Ethical approval was obtained from Teesside University School of Health \& Social Care Research Governance and Ethics Committee (Study No. 116/18).

A qualitative descriptive approach was selected to answer the research questions, as it is the favourable method to use when an intervention needs to be developed based on the beliefs and expectations of a group of individuals (18). It is also helpful in understanding a phenomenon from the participant's everyday perspective (19). Focus group discussions were used to obtain data, as it facilitates a discussion among participants who share similar attributes but possess divergent views, which helped to achieve the objectives of the research (20).

\section{Participant recruitment and inclusion criteria}

\section{Inclusion criteria}

Pregnant or postpartum ( $<1$ year after delivery) women who were active during pregnancy or were already active before pregnancy were considered eligible for inclusion in the study. Women were considered active if they self-reported a minimum of 150 minutes of moderate to vigorous physical 
activity (MVPA) per week, which was confirmed by asking the participants to acknowledge this in the consent form.

\section{Recruitment}

Participants were recruited from the Durham and Darlington areas of Northern England in the UK. These areas have the lowest sport and physical activity participation levels in the UK(21) and large healthinequalities (22). Participants were initially recruited using posters and flyers at the Children's Centres in the target area, where regular sessions for antenatal and postnatal women were conducted. However, as this recruitment method was unsuccessful, and no participant enquiry was received, recruitment was expanded to include presentations at reading clubs for pregnant women and word of mouth. A total of 19 participants expressed interest in participating and met the inclusion criteria, and all were included in the study. Signed informed consent was obtained from all participants. Five focus group discussions were used to obtain data.

\section{Data Collection}

All focus groups were conducted within Children's Centres, and a topic guide was used to guide discussion around the project objectives (Additional file 1).

All focus groups were conducted by MS (a doctoral student and physician) who had no professional contact with any of the participants before the study. The focus groups lasted between 70 and 95 minutes. After the completion of four focus groups $(n=15)$, data saturation was achieved, and no new themes emerged. However, a fifth focus group took place as it had already been arranged with the participants. This focus group confirmed previous findings.

\section{Data Analysis}

The focus group sessions were audio-recorded using a digital recorder (Victure-V6) and transcribed verbatim. All person identifiers were removed during transcription.

An iterative approach was used for data collection and analysis. Two of the authors (MS and LA) met regularly to discuss the focus group findings and interpretation. An inductive thematic analysis was carried out on the focus group data (23) by MS, who coded all the transcripts. Four study authors (LA, PG, RD, KS) coded a subset of transcripts (60\%) and (MS, LA and PG) met to discuss any discrepancies which were resolved by adjudication (discussion between all three researchers). The codes applied to all the transcripts were summarised and assigned to broader themes.

\section{Study Quality}

The consolidated criteria for reporting qualitative research (COREQ) checklist (24) was used to ensure the findings are reported in a complete and transparent manner, thereby ensuring the rigour and credibility of the research. Reflective notes were maintained by MS after each focus group. This informed the better conduct of the remaining focus group sessions and also helped form the codes and themes. 


\section{Results}

The five focus group discussions ( $\mathrm{n}=19)$ were conducted between December 2018 and January 2019. Out of the 19 participants, four were postpartum, and 15 were pregnant. Mean age of the pregnant and postpartum participants was $28.4 \pm 3.17$ years and $28.8 \pm 2.04$ years, respectively. All participants were White, British and spoke English as their first language. The mean gestational age among pregnant participants was $26.8 \pm 4.49$ weeks, and the mean age of the children from the postpartum women was 5 \pm 1.41 months. Physical activities undertaken by the participants included cycling, swimming, football and circuit training. Eight overarching themes were identified from the transcripts of the five focus groups, of which six themes directly related to already active women's perceptions of barriers and facilitators of physical activity during pregnancy, and two themes related to the women's requirements for a physical activity intervention.

\section{Identified themes}

\section{Cognizant of Physical Activity Benefits}

Almost all the participants were aware of the current guidelines that are available for physical activity in adults (25). However, the women were not aware of a separate guideline for physical activity during pregnancy.

...I think it's only for England that the government's recommendations are 150 minutes of physical activity...[P1]

...should include every type of activity, and can't restrict with just aerobics or muscle training...[P18]

... do they have something specific for us? I don't think, there is.....[P7]

While the women participated in the focus groups were aware of the recommendations and guidelines, they were also appreciative of the health benefits that an active lifestyle offers, including mental wellbeing.

...I know that there a lot of advantages and perks that it has and it brings to you...[P3]

...it gave me a sense of well being so I felt good doing physical activity...[P7]

Other benefits of physical activity, such as having fun, were also highlighted. A couple of participants commented on the benefits of joint physical activity with their partners, which helped in strengthening the relationships and bonds between them. 
... really boosts the bond between me and my partner and it's also really helpful for us to keep keep ourselves fit...[P4]

\section{Sources of Advice}

Almost all the study participants credited midwives as their source of advice when it came to doing physical activity during and after pregnancy. They had regular conversations regarding the amount, mode and safety of exercise in and around pregnancy, and found the resources that were given to them by their midwives, to be valuable. Another important source of information were websites and online forums, where women receive peer support and advice from other women who had real-time experiences that they share in the discussion forums.

... My midwife has a lot of handouts or pamphlets which also gives loads of info...[P12]

... [mentioned website] is one source that that's where we could find many online forums for physical activity and pregnant women...[P3]

\section{Reasons to be active during pregnancy}

During the focus group discussions, the study participants were encouraged to share their own reasons to be active during pregnancy. Women felt that doing exercise was "not going to harm" and thought that they should continue to receive the benefits for themselves and the baby.

... I felt I should be physically active and keep myself and the growing baby healthy, and it's not gonna cause any harm...[P2]

Although they feel that exercise and physical activity during pregnancy is safe, most of the study participants were of the opinion that there is a "safe level" which differs from person to person. They tend to avoid the same intensity and level of activities that they were doing before pregnancy.

... I did reduced it to half mainly because I was very concerned about the safety of myself and the kid or the baby...[P1]

\section{Barriers to Physical Activity During pregnancy}


All of the study participants who were pregnant believed that their "growing tummy" is a substantial barrier for being active during pregnancy. As pregnancy progresses, the size of their growing abdomen poses difficulties in carrying out certain activities such as running, thereby they should reduce the amount and type of exercise during advanced pregnancies.

... I felt my bump, my tummy is getting bigger and I thought I should reduce it as it is hard...[P1]

Another key barrier that was highlighted by the study participants was that they felt short of breath when they tried to do the same amount of activities as earlier, and they use this as a marker or cut-off point for stopping their exercise. The participants perceived higher intensity exercise as unsafe for the baby. Therefore, shortness of breath which occurs at more intense exercise, was perceived as unsafe.

... I eventually I could not do a lot as I become breathless at times, so I stick onto...[P16]

... At times I may stop my exercise panting heavily, and know that's when I should stop....[P7]

... I know when to stop, when I start panting, I know its not good for the baby and I stop...[P11]

\section{Barriers to physical activity after pregnancy}

Postpartum women in the study had a different set of barriers, with childcare at the top of the list. They felt that arranging childcare was difficult, expensive and nonessential, especially when they are spending for both the childcare and their exercise or activity. The study participants obviously believe that it is essential to be physically active, but consider that in balancing childcare costs and difficulty, it would make exercising not a priority.

...I have to take care of my boy and childcare is not too easy these days...[P16]

...nothing can stop me, but I fear the costs of childcare will cause big troubles...[P17]

.../ agree, spending money on childcare, and again spend some money to swim is definitely not in my court...[P16]

Another related barrier highlighted by the study participants is that they "do not want to spend any time away from baby". They consider that it is the time to stay with the newborn and develop their bond rather than spending time away from the baby for exercise and physical activity.

...is the time to cherish, I don't want to lose those precious time with my baby...[P17] 
Fatigue seems to be another key barrier in postpartum women, preventing them from being active. The study participants cited getting tired quickly, especially with the baby around as a barrier for resuming their pre-pregnancy activities.

...my lack of energy makes me think I won't be fit anytime soon to come back to my previous levels...[P19] ...I get tired easily, and I think because I feed my baby I become weak...[P18]

\section{Facilitators of physical activity during and after pregnancy}

Access to activities and facilities that are exclusively available for already active women during pregnancy is often highlighted as an essential facilitator to maintain their activity levels during and after pregnancy. Participants also felt that, although such activities may exist, they are not available everywhere, and the participants were not aware of them.

...however, don't think I can do the same exercise and activities that I did earlier, I need something specific that can be done in pregnancy...[P6]

...my mom always use to say, don't do the same when you are pregnant. But, I didn't know anything else... [P14]

...it is a shame, that this part of the country is always neglected, I know many places in the south that has a lot of gyms and studios exclusive to pregnant women. [P12]

The study participants considered it helpful to have group activities for women who are either pregnant or have a newborn. They felt the opportunity to share their experiences and receive peer support would encourage attendance.

...it will be fun if you can do it as a group where we can share our experiences...[P5]

...it would definitely motivate and encourage when we do it as a big group of women who are pregnant or have babies...[P11]

The participants also highlighted that they feel safe and comfortable to do an activity that has already been carried out by other pregnant and postpartum women and found to be safe. 
...it is better to do what you and others were already doing especially when you know they are safe and time tested...[P13]

\section{Ideal Physical Activity intervention}

The study participants came up with a number of suggestions when the discussion focused on the prerequisites of a physical activity intervention that would be designed to assist already active women and help them maintain or increase their activity levels during and after pregnancy. There was a mixed response as to whether the intervention must have the usual activities that they already do or could include new activities for them to try.

...it gives you confidence to do when you do something you are already used to...[P15]

... When you know it's done by many, I do not see any issues trying out new activities...[P10]

... at times I am bored... why not try to do some exciting new stuff...[P19]

Though there is a difference of opinion as to whether to use the same or new activities in the programme, there was consensus among the participants that, any activity should provide the same amount of social and activity-wise 'satisfaction and fun' as the previous activities offer.

...need something that when done gives me the sense of satisfaction...[P3]

...usually feel so happy after swimming, and would like to feel the same or even more after any new thing I do...[P14]

Study participants suggest that the activity must be offered near their residences, as they feel it will be a "hassle" for them to travel, especially when it is a new venue.

...definitely will be a hassle to drive even if it is just a few miles...[P8]

...can't trust it when the place is new and far...[P13]

\section{Evaluation support}

Participants did not want to wear any type of activity tracking device in order to monitor their activity levels. They felt that such wearable devices would make the activity inconvenient and less fun. However, 
the participants also commented that if the devices are smaller and fashionable like wristwatches, they would be happy to use them.

...it will be inconvenient, and defeats the sense of joy I get from exercise...[P5]

...if it is like Fitbit or something like that, it is alright with me...[P13]

Although they were not comfortable about wearing activity tracking devices, almost all the study participants were happy about filling out brief questionnaires in order to help the evaluation.

...don't mind filling out forms...[P4]

...they should be brief though... [P6]

\section{Discussion}

The current study aimed to explore the facilitators and barriers to physical activity in already active women during pregnancy and postpartum and gather information on the design of a tailored intervention to this population. Similar to previous findings, this study demonstrated that already active women were willing to continue their physical activity throughout pregnancy and postpartum (17), and the participants showed a clear understanding of the benefits of being active during and after pregnancy. Pregnant women consider their changing body physique and fatigue as a significant barrier, while postpartum women feel that taking care of the child prevents them from being active. However, both groups consider exercise during and after pregnancy, safe and beneficial at a certain level.

An interesting finding that was highlighted in this focus group study was that already active pregnant and postpartum women expect an ideal physical activity intervention to have activities that are both familiar and new but offering the same satisfaction of doing a substantial physical activity as the activities they use to do before pregnancy. Likewise, women are unwilling to travel in order to participate in such activities; therefore, activity provision in the neighbourhood might be an important facilitator. In agreement with this, some previous interventions identified in the literature have been successful in increasing the activity levels of already active pregnant and postpartum women by facilitating easyaccess to sessions. For example, a provision in the same venue as they receive antenatal care; using mobile applications, DVDs and online links containing pregnancy-specific dietary advice and exercises, and including continued goal setting and monitoring tasks $(26,27)$.

Women that have already been active before pregnancy have a good awareness of the benefits of physical activity. This finding is consistent with the literature on active women's leisure-time physical activity (LTPA), where studies have confirmed that pregnant women perceive the benefits associated with MVPA and LTPA $(28,29)$. However, women tend to reduce physical activity levels during pregnancy because of their beliefs of potential danger with their exercise levels and durations.(30) Contrarily, the 
current study has observed that almost half of the active women included in the study perceive physical activity to be safe to both the women and foetus. Study participants were aware of body changes that limit the physical activities they can do safely, but they simply adjusted their activities or intensity level, and particularly avoided higher intensity exercise, judged by shortness of breath.

The difference between this and the previous study (30), may be attributable to the fact that the participants in the current study had an active lifestyle before pregnancy and hence see the potential benefits of being active, thereby reaffirming that a tailormade physical activity intervention is needed to this group of already active pregnant and postpartum women.

Similarly, societal pressures from peers on the safety of the women and foetus while doing physical activity during pregnancy are not considered as a barrier among the study participants. In contrast, previous studies have highlighted this as a significant factor in preventing non-active women from engaging in physical activity during and after pregnancy $(31,32)$. This again may be attributed to the prepregnancy lifestyle of the study participants, wherein they were regarded active by their social networks, and hence continuing their physical activity into pregnancy is not considered different.

Findings from this study might also support the development of a tailored intervention to active women. Women in this study had a mixed reaction regarding the type of activities to be included. As expected from a group of women who participate in a wide range of activity before pregnancy, no single activity could be pinpointed, but all women felt that any activity included should be social and activity-wise 'fun and satisfying'. As a consensus, the participants in the study agreed that the intervention for active women during pregnancy should consist of a mixture of both usual physical activities and newer ones, along with social elements and activities in which they can include their family members. Also, the participants were interested in having the activities delivered closer to their residences and during times that would suit them generally. Another key expectation from postpartum women in the study is the possibility of having childcare at the same venue where the intervention is being delivered or to have activities that incorporate the newborn.

Although several studies explore the beliefs, barriers and enablers of physical activity during and after pregnancy $(16,28,30,33)$, little has been studied on the beliefs of pregnant and postpartum women who were already active (17). A similar qualitative research study on this topic was carried out on participants 3 to 4 years postpartum, where they shared their memories of experiences during pregnancy (17). The current study included pregnant women and women within one year of delivery, thereby the information obtained is current and relevant and extend the evidence regarding active pregnant and post-pregnant women's beliefs on physical activity. In addition, this study has provided critical insights, such as the components of a tailored intervention to help increase their activity levels.

However, findings from the current study must be generalised with caution, as it has some limitations. The focus groups were all conducted within a defined location in the northeast of England and among white women. This limits the generalisability of study findings, and hence in future, this should be explored among culturally diverse groups in a wider geographical area. Among the study participants, 
pregnant women were in more significant number in comparison to postpartum women (15 pregnant women and four postpartum). Although both groups were already active before pregnancy, their psychological, physiological and anatomical status pre and post-delivery were different and hence their opinion on the facilitators and barriers of physical activity. However, we tried to differentiate this in the results and discussion. Another limitation of the study is that the first author (MS) conducted all the focus groups, thereby increasing the possibility of observer bias (34). However, the author tried to mitigate this by encouraging active participation from all the study participants while limiting the interviewer interaction (35). Another limitation in the study is that meeting the inclusion criteria of a minimum of 150 minutes of MVPA per week was self-reported by the participant rather than objectively measured. However, the main author (MS) provided a clear explanation to participants on what activities could be counted as moderate or vigorous, to reassure that they meet the inclusion criteria. This also became evident through their discussion during the focus group, where women reinforced that they were highly active and perform athletic level activities.

\section{Conclusions}

In summary, the current study shows that already active women believe exercise is beneficial during and after pregnancy. They also consider it to be essential to promote health benefits and for personal satisfaction. A significant barrier preventing physical activity in pregnant women is changing body shape, while for postpartum women is the need for childcare. Interventions attempting to increase activity levels in already active women during pregnancy and postpartum must be designed with a comprehensive approach to include both old and new activities. Further, such interventions must aim to offer the same if not more social 'satisfaction and fun', that the pre-pregnancy activities offered.

The findings from this focus group study will inform the development of a physical activity intervention during pregnancy and postpartum for already active women as it enables us to understand their expectations and needs. This novel intervention will be piloted in deprived communities in the North East of England, in partnership with a local community organisation, as part of a larger project, the Sport England - Helping the active to Stay Active, which has the overarching aim of informing the design of a physical activity intervention for currently active pregnant and postpartum women.

Similar studies should be conducted in different geographical locations and among culturally diverse groups, which presents different levels of physical activity (36).

\section{List Of Abbreviations}

LTPA - Leisure-time physical activity

MVPA - Moderate to vigorous physical activity

PA - Physical activity

Page 12/16 


\section{Declarations}

\section{Ethics Approval and Consent to Participate}

Ethical approval was obtained from Teesside University School of Health \& Social Care Research Governance and Ethics Committee (Study No. 116/18). Signed informed consent was obtained from participants included in the study.

\section{Consent for Publication}

Written informed consent was obtained from the participants for publication of direct quotes from focus groups in this manuscript. The consent form is held by the author and is available for review by the Editor-in-Chief.

\section{Availability of Data and Materials}

The datasets generated and/or analysed during the current study are not publicly available due to the fact that it contains participant's private and confidential information but are available from the corresponding author on reasonable request.

\section{Competing Interests}

The authors declare that they have no competing interests.

\section{Funding}

Dr Peter Van Der Graaf and Dr Louise Hayes are members of Fuse, the Centre for Translational Research in Public Health (www.fuse.ac.uk). Fuse is a UK Clinical Research Collaboration (UKCRC) Public Health Research Centre of Excellence. Funding for Fuse from the British Heart Foundation, Cancer Research UK, National Institute of Health Research, Economic and Social Research Council, Medical Research Council, Health and Social Care Research and Development Office, Northern Ireland, National Institute for Social Care and Health Research (Welsh Assembly Government) and the Wellcome Trust, under the auspices of the UKCRC, is gratefully acknowledged. The views expressed in this paper do not necessarily represent those of the funders or UKCRC. The funders had no role in study design, data collection and analysis, decision to publish, or preparation of the manuscript. This study was also partly funded by a grant from Sport England as part of the PhD studentship for MS.

\section{Authors' Contributions}

MS and LA conceived and designed the study. MS collected all the data. MS, LA, RD, PG and KS were involved in analysing the data. MS drafted the manuscript. LA, LE, LH and PG advised in the design of the study, data collection, analysis and interpretation of findings as well as critically commenting on the draft manuscript. All authors read and approved the final manuscript. 
Not Applicable

\section{References}

1. Hopkins SA, Cutfield WS. Exercise in pregnancy: weighing up the long-term impact on the next generation. Exerc Sport Sci Rev. 2011;39(3):120-7.

2. Leite CF, do Nascimento SL, Helmo FR, dos Reis Monteiro MLG, dos Reis MA, Corrêa RRM. An overview of maternal and fetal short and long-term impact of physical activity during pregnancy. Archives of gynecology obstetrics. 2017;295(2):273-83.

3. Nascimento SL, Surita FG, Cecatti JG. Physical exercise during pregnancy: a systematic review. Curr Opin Obstet Gynecol. 2012;24(6):387-94.

4. Ramírez-Vélez R, Aguilar de Plata AC, Escudero MM, Echeverry I, Ortega JG, Salazar B, et al. Influence of regular aerobic exercise on endothelium-dependent vasodilation and cardiorespiratory fitness in pregnant women. Journal of Obstetrics Gynaecology Research. 2011;37(11):1601-8.

5. Barakat R, Cordero Y, Coteron J, Luaces M, Montejo R. Exercise during pregnancy improves maternal glucose screen at 24-28 weeks: a randomised controlled trial. Br J Sports Med. 2012;46(9):656-61.

6. Oken E, Ning Y, Rifas-Shiman SL, Radesky JS, Rich-Edwards JW, Gillman MW. Associations of physical activity and inactivity before and during pregnancy with glucose tolerance. Obstetrics gynecology. 2006;108(5):1200.

7. Sanabria-Martínez G, García-Hermoso A, Poyatos-León R, González-García A, Sánchez-López M, Martínez-Vizcaíno V. Effects of exercise-based interventions on neonatal outcomes: a meta-analysis of randomized controlled trials. American Journal of Health Promotion. 2015:ajhp. 140718-LIT-351..

8. Scholes S, Neave A. Health Survey for England 2016: Physical activity in adults. Health. \& Social Care Information Centre Retrieved from http://www hscic gov uk/catalogue/PUB13218/HSE201. 2013.

9. Melzer K, Schutz Y, Boulvain M, Kayser B. Physical activity and pregnancy. Sports Med. 2010;40(6):493-507.

10. Evenson KR, Wen F. Prevalence and correlates of objectively measured physical activity and sedentary behavior among US pregnant women. Preventive medicine. 2011;53(1-2):39-43.

11. Borodulin K, Evenson KR, Wen F, Herring AH, Benson A. Physical activity patterns during pregnancy. Med Sci sports Exerc. 2008;40(11):1901.

12. Fell DB, Joseph $\mathrm{K}$, Armson BA, Dodds $\mathrm{L}$. The impact of pregnancy on physical activity level. Matern Child Health J. 2009;13(5):597.

13. Gaston A, Cramp A. Exercise during pregnancy: a review of patterns and determinants. Journal of Science Medicine in Sport. 2011;14(4):299-305. 
14. Clarke P, Rousham E, Gross H, Halligan A, Bosio P. Activity patterns and time allocation during pregnancy: a longitudinal study of British women. Ann Hum Biol. 2005;32(3):247-58.

15. Albright CL, Maddock JE, Nigg CR. Physical activity before pregnancy and following childbirth in a multiethnic sample of healthy women in Hawaii. Women Health. 2006;42(3):95-110.

16. Evenson KR, Aytur SA, Borodulin K. Physical activity beliefs, barriers, and enablers among postpartum women. Journal of women's health. 2009;18(12):1925-34.

17. Hegaard HK, Kjaergaard H, Damm PP, Petersson K, Dykes A-K. Experiences of physical activity during pregnancy in Danish nulliparous women with a physically active life before pregnancy. A qualitative study. BMC Pregnancy Childbirth. 2010;10(1):33.

18. Sullivan-Bolyai S, Bova C, Harper D. Developing and refining interventions in persons with health disparities: The use of qualitative description. Nurs Outlook. 2005;53(3):127-33.

19. Sandelowski M. Whatever happened to qualitative description? Research in nursing \& health. 2000;23(4):334-40.

20. Powell RA, Single HM. Focus groups. International journal for quality in health care. 1996;8(5):499504.

21. INACTIVE: LESS THAN 30 MINUTES A WEEK - ACTIVE LIVES SURVEY NOV 17/18 England England S. LEVELS OF ACTIVITY -. INACTIVE: LESS THAN 30 MINUTES A WEEK - ACTIVE LIVES SURVEY NOV 17/18 England: Sport England; 2020 [Available from:

https://activelives.sportengland.org/Result?queryld=13983.

22. Bambra C, Barr B, Milne E. North and South: addressing the English health divide. Oxford University Press; 2014.

23. Braun V, Clarke V. Using thematic analysis in psychology. Qualitative research in psychology. 2006;3(2):77-101.

24. Tong A, Sainsbury P, Craig J. Consolidated criteria for reporting qualitative research (COREQ): a 32item checklist for interviews and focus groups. International journal for quality in health care. 2007;19(6):349-57.

25. UK physical activity guidelines: GOV.UK; 2020 [Available from: https://www.gov.uk/government/publications/uk-physical-activity-guidelines.

26. Kennelly MA, Ainscough K, Lindsay KL, O'Sullivan E, Gibney ER, McCarthy M, et al. Pregnancy exercise and nutrition with smartphone application support: a randomised controlled trial. Obstetrics Gynecology. 2018;131(5):818-26.

27. Kominiarek MA, Vyhmeister H, Balmert LC, Fairchild P, Tolo H, Grobman W, et al. Activity tracking devices in group prenatal care: a feasibility study. BioResearch open access. 2018;7(1):165-76.

28. Evenson KR, Bradley CB. Beliefs about exercise and physical activity among pregnant women. Patient Educ Couns. 2010;79(1):124-9.

29. Da Costa D, Ireland K. Perceived benefits and barriers to leisure-time physical activity during pregnancy in previously inactive and active women. Women Health. 2013;53(2):185-202. 
30. Clarke PE, Gross H. Women's behaviour, beliefs and information sources about physical exercise in pregnancy. Midwifery. 2004;20(2):133-41.

31. Kieffer EC, Willis SK, Arellano N, Guzman R. Perspectives of pregnant and postpartum Latino women on diabetes, physical activity, and health. Health Education Behavior. 2002;29(5):542-56.

32. Leiferman J, Swibas T, Koiness K, Marshall JA, Dunn AL. My baby, my move: examination of perceived barriers and motivating factors related to antenatal physical activity. Journal of midwifery women's health. 2011;56(1):33-40.

33. Weir Z, Bush J, Robson SC, McParlin C, Rankin J, Bell R. Physical activity in pregnancy: a qualitative study of the beliefs of overweight and obese pregnant women. BMC Pregnancy Childbirth. 2010;10(1):18.

34. Patton MQ. Qualitative research. Encyclopedia of statistics in behavioral science. 2005.

35. Stiles WB. Quality control in qualitative research. Clin Psychol Rev. 1993;13(6):593-618.

36. Koshoedo S, Simkhada P, Van Teijlingen E. Review of barriers to engaging black and minority ethnic groups in physical activity in the United Kingdom. Global Journal of Health Science. 2009;1(2):8596.

\section{Supplementary Files}

This is a list of supplementary files associated with this preprint. Click to download.

- AdditionalFile1.docx 